\title{
Developmental and cAMP-mediated regulation of glycogen phosphorylase 1 in Dictyostelium discoideum
}

\author{
Joseph F. Sucic, $\uparrow$ Shun Luo $\ddagger$ Brian D. Williamson, Yizhong Yin, Patricia V. Rogers \\ and Charles L. RutherforD*
}

Biology Department, Cellular and Molecular Biology Section, Virginia Polytechnic Institute and State University, Blacksburg, VA 24061, USA

(Received 5 April 1993; revised 11 June 1993; accepted 23 June 1993)

\begin{abstract}
The Dictyostelium discoideum glycogen phosphorylase-1 (gp-1) exhibits a complex pattern of developmental expression in which differential temporal regulation of enzyme activity, protein levels and mRNA levels is observed. This pattern of expression implies that gp-1 regulation occurs at multiple levels, probably involving both transcriptional and post-transcriptional events. Post-translational control of gp-1 activity, in effect, actually regulates the protein from a developmental perspective. In this report we have examined several facets of this regulation. We show that addition of exogenous cAMP to cells in suspension culture caused changes in gp-1 enzyme activity and mRNA levels that are identical to those observed during normal development, suggesting that cAMP is involved in the regulation of gp-1. Exogenous cAMP could regulate gp-1 mRNA expression at concentrations as low as $1.0 \mu \mathrm{M}$. cAMP regulation of gp-1 mRNA appeared to occur through a mechanism that required intracellular cAMP signalling. We identified regions of the promoter necessary for gp-1 expression by using gp-1 promoter deletions to drive the expression of a luciferase reporter gene. Results of these experiments suggested that developmental and cAMP-mediated changes in gp-1 mRNA levels were the result of alterations in transcription. The promoter analysis also suggested that a vegetative specific element is located between -785 and -1894 nucleotides from the transcriptional start site. Elements necessary for maximal developmental and cAMPmediated expression appear to be located between -1153 and -1894 nucleotides from the cap site. Sequence elements located between $-\mathbf{1 8 0}$ and -1153 appear to be required for a basal level of late developmental expression.
\end{abstract}

\section{Introduction}

One of the central problems in biology is the elucidation of the molecular control mechanisms responsible for

*Author for correspondence. Tel. + 17032315349 ; fax +1703231 9307.

$\dagger$ Present address: Department of Microbiology and Molecular Genetic and Markey Center for Molecular Genetics, University of Vermont, Burlington, VT 05405, USA.

$\ddagger$ Present address: Dana Farber Cancer Institute, Boston, MA 02115, USA.

Abbreviations: $\mathrm{Bt}_{2}-\mathrm{cAMP}$, dibutyryl-cAMP; 2'd-cAMP (2'-deoxycAMP); gp-1, glycogen phosphorylase-1; gp-2, glycogen phosphorylase-2; SSPE $(1 \times), 8.77 \mathrm{~g} \mathrm{NaCl}, 1.38 \mathrm{~g} \mathrm{NaH}_{2} \mathrm{PO}_{4} \cdot \mathrm{H}_{2} \mathrm{O}$, $0.37 \mathrm{~g}$ EDTA made to 1 litre after bringing the $\mathrm{pH}$ to 7.4 with $\mathrm{NaOH}$..

The GenBank accession number for the sequence reported in this paper is X62142. developmentally regulated gene expression. In recent years it has become increasingly clear that the expression of developmentally regulated genes can be controlled at many different levels; transcriptional control, mRNA processing and stability, translational control and posttranslational protein modification have all been shown to play roles in regulating the developmental expression of various genes.

The cellular slime mould Dictyostelium discoideum provides an ideal model system to study the molecular events associated with development and cell differentiation (for a review see Loomis, 1982). This organism proceeds through a short and well-documented developmental cycle that is initiated by nutrient depletion. Upon nutrient depletion, vegetative cells, which are amoeboid in appearance, halt cell division, stream together, and ultimately form a multicellular fruiting structure composed of two distinct cell types. This developmental cycle not only possesses elegant sim- 
plicity, but also separates vegetative growth from differentiation (Sussman \& Brackenbury, 1976). Additionally, Dictyostelium is readily amenable to the genetic manipulations required for modern molecular biology techniques.

Both differentiated cell types in Dictyostelium require the synthesis of complex structural polysaccharides such as cellulose, and the glucose monomers used to synthesize these structural components are derived from glycogen stores (Wright et al., 1968; Marshall et al., 1970; Gustafson \& Wright, 1972). Thus, glycogen degradation is a crucial developmental event in Dictyostelium. Glycogen degradation is catalysed by glycogen phosphorylase $\quad(1,4-\alpha$-D-glucan:orthophosphate $\alpha$-D-glucosyltransferase, EC 2.4.1.1), and our laboratory is examining the expression of two developmentally regulated glycogen phosphorylases in Dictyostelium. Glycogen phosphorylase-2 (gp-2) is expressed late in development, and the gp-2 mRNA and protein accumulate concomitantly with increases in gp-2 activity (Naranan et al., 1988; Rutherford et al., 1992). Glycogen phosphorylase-1 (gp-1) exhibits a more complex pattern of regulation. gp-1 enzyme activity is maximal in vegetative amoebae, then decreases throughout the subsequent stages of development (Rutherford \& Cloutier, 1986; Naranan et al., 1988). Although the gp1 activity decreases, the level of gp-1 protein is constant during development (Naranan et al., 1988; Rogers et al., 1992). The gp-1 mRNA also exhibits developmental regulation (Rogers et al., 1992). In vegetative amoebae and in very early developmental stages, the gp-1 mRNA is highly expressed, but as development proceeds the level of gp-1 mRNA decreases; later in development, gp1 mRNA levels increase again. The differential temporal expression of gp-1 enzyme activity, protein and mRNA suggest that regulation of gp-1 occurs at multiple levels, possibly involving both transcriptional and posttranscriptional events. In this report we examine several aspects of gp-1 regulation. We show that exogenous cAMP can mimic the developmental changes seen in gp1 enzyme activity and mRNA levels. We also examine the molecular mechanism of cAMP regulation of gp-1 and examine the gp-1 promoter to identify regions necessary for developmental and cAMP-mediated expression. The patterns of gp-1 RNA expression, protein levels and enzyme activities indicate that posttranslational regulation is most important from a developmental perspective.

\section{Methods}

Materials. Radiolabelled $\left[{ }^{32} \mathrm{P}\right] \mathrm{dATP}$ was obtained from New England Nuclear. Ultrapure phenol was from Bethesda Research Laboratories. Nitrocellulose (Nitro Plus 2000) was obtained from MSI. Unless otherwise indicated all other reagents were obtained at the highest purity available from Sigma.

Cell culture. Vegetative cells of Dictyostelium discoideum strain AX3 were cultured in HL5 as described previously (Naranan et al., 1988; Rogers et al., 1992). Dictyostelium strain AX3K was used for the reporter gene experiments. The $\mathrm{AX} 3 \mathrm{~K}$ cells were kept at a density of less than $5.0 \times 10^{6} \mathrm{ml}^{-1}$, and were used at a density of $1.0 \times 10^{6} \mathrm{ml}^{-1}$ in the transformation protocol. To obtain cells at various developmental stages, vegetative amoebae were washed free of nutrient media and plated onto Gelman GN-6 cellulose acetate filters supported by Gelman absorbent pads saturated with MES/LPS buffer ( 7 mM-MES/ $\mathrm{NaOH}$ pH 6.5, 5 mm-MgSO ${ }_{4}, 20$ mM-KCl).

cAMP response experiments. Dictyostelium amoebae were made aggregation-competent by plating onto non-nutrient agar $(1.5 \%, \mathrm{w} / \mathrm{v}$, Bacto-agar buffered at $\mathrm{pH} 6.5$ by $7 \mathrm{~mm}$-MES) and incubating for $12 \mathrm{~h}$ at $7{ }^{\circ} \mathrm{C}$. The aggregation-competent cells were then harvested, washed once in MES/LPS, and resuspended in $30 \mathrm{ml}$ fresh MES/LPS at $0.5-1 \cdot 0 \times 10^{6}$ cells $^{-1}$ in $125 \mathrm{ml}$ Erlenmeyer flasks. cAMP was added to a final concentration of $1.0 \mathrm{~mm}$ and the cells were shaken at 180 r.p.m. on a Lab Line rotary shaker (model 3250). Samples of the cell suspension were removed at $4 \mathrm{~h}$ and/or $8 \mathrm{~h}$ after cAMP addition for use in various assays. When $8 \mathrm{~h}$ cells were used, the cells were pelleted by centrifugation after $4 \mathrm{~h}$ shaking and resuspended in fresh MES/LPS with or without added cAMP. The analogue experiments were carried out as above except that $2.0 \mathrm{~mm}$-caffeine was added to the MES/LPS prior to the addition of either $1.0 \mathrm{~mm}-2^{\prime}$-deoxy-cAMP ( $2^{\prime} \mathrm{d}$-cAMP) or 1.0 mM-dibutyryl-cAMP ( $\mathrm{Bt}_{2}$-cAMP).

Glycogen phosphorylase activity assays and Western blotting. Cell extracts from cAMP shaking experiments or developmental studies were prepared by freeze-thaw lysis, and a $20 \mu$ sample of each extract was added to $200 \mu \mathrm{l}$ of a reaction mixture containing $50 \mathrm{~mm}$-imidazole (pH 6-8), $2.5 \mathrm{mg}$ glycogen $\mathrm{ml}^{-1}, 2 \mathrm{~mm}-\mathrm{K}_{2} \mathrm{HPO}_{4}, 5 \mathrm{~mm}-\mathrm{MgCl}_{2}, 0.5 \mathrm{~mm}-$ NADP, $50 \mu \mathrm{g}$ glucose 1,6-bisphosphate $\mathrm{ml}^{-1}, 0.3$ units glucose-6phosphate dehydrogenase and 0.4 units phosphoglucomutase. The assay was carried out at $23^{\circ} \mathrm{C}$. The amount of NADPH formed was followed on a strip chart recorder. A molar absorption coefficient of $6.2 \times 10^{3}$ litre $\mathrm{mol}^{-1} \mathrm{~cm}^{-1}$ was used to quantify NADPH formation at $350 \mathrm{~nm}$. One unit (U) of activity is the amount of enzyme that catalyses the synthesis of $1 \mu \mathrm{mol} \mathrm{NADPH} \mathrm{min}^{-1}$ at $23^{\circ} \mathrm{C}$.

For Western blotting, proteins were resolved by SDS-PAGE and electrophoretically transferred to nitrocellulose membranes using a Hoeffer transfer chamber and a power source (model TE51) set at $1.2 \mathrm{~A}$ for $1 \mathrm{~h}$. The transfer buffer contained $192 \mathrm{~mm}$-glycine and $20 \%(\mathrm{v} / \mathrm{v})$ methanol in $25 \mathrm{~mm}$-Tris $/ \mathrm{HCl}, \mathrm{pH} 8.3$. After transfer, the visualization of gp-1 protein was done as previously described (Naranan et al., 1988), using polyclonal anti-gp-1 antibodies, a protein-A-peroxidase conjugate, and colorimetric antigen detection with 4-chloro-1-naphthol and hydrogen peroxide.

RNA isolation and Northern blot analysis. Total RNA was prepared from Dictyostelium cells by the simultaneous lysis and extraction in diethylpyrocarbonate (DEPC), SDS and phenol/chloroform as described previously (Nellen et al., 1987). Northern blotting was done by capillary action following gel electrophoresis of the RNA in formaldehyde containing $1.2 \%(\mathrm{w} / \mathrm{v})$ Seakem (FMC) agarose. RNA was quantified by optical density reading at $260 \mathrm{~nm}$, and $10 \mu \mathrm{g}$ of total RNA was loaded per lane. Duplicate samples were run and stained with ethidium bromide to ensure that rRNA bands were of equal intensity in all lanes. Hybridizations were performed under stringent conditions of $50 \%(\mathrm{v} / \mathrm{v})$ formamide, $6 \times$ SSPE, $1 \times$ Denhardt's solution, $0.1 \%$ SDS and $200 \mu \mathrm{g}$ salmon sperm DNA at $42{ }^{\circ} \mathrm{C}$. Purified cDNA fragments of either 1421 bp or 836 bp (Rogers et al., 1992) were labelled by random priming (Feinberg \& Vogelstein, 1983) and used in the hybridizations. Hybridized filters were washed in $0.2 \times \mathrm{SSC}, 0.2 \%$ 
SDS at $42-65^{\circ} \mathrm{C}$. The hybridized filters were dried under vacuum and autoradiography was done by exposing the filters to Kodak XAR-5 film at $-70{ }^{\circ} \mathrm{C}$.

Construction of the gp-l promoter deletions. A $2.2 \mathrm{~kb} R s a \mathrm{I}$ genomic fragment containing approximately 200 nucleotides of transcribed sequence and $2.0 \mathrm{~kb}$ of $5^{\prime}$ sequence was isolated and inserted into the SmaI site of the vector pBluescript II SK + . Deletions of the promoter region were prepared by digesting the vector at the $S a c \mathrm{I}$ site, filling with deoxythioderivatives using the Klenow fragment of $E$. coli DNA polymerase I, and treating with Exo III and mung-bean nucleases for various times. After ligation and transformation of bacteria, recombinant clones were selected and sequenced.

Ligation of the promoter deletions into the reporter gene vector. The promoter inserts were cut out of pBluescript with ClaI/BssHII double digests and were blunt-ended by filling in overhangs with the Klenow enzyme. The vector SP60-luciferase- $\Delta 20$ (SP60-luc; see Haberstroh \& Firtel, 1990) was the generous gift of Richard A. Firtel from the University of California, San Diego, USA. The SP60 promoter was excised from this vector with a NdeI/ClaI double digest, and the overhangs were filled in. The gp-1 promoter inserts contain the translation initiation codon, so ligations were constructed to allow the in-frame read through from the gp-1 initiation codon, through a short stretch of pBluescript sequence, and into the luciferase structural gene. Ligations were done with T4 DNA ligase (BMB) according to the supplier's instructions. The molar ratio of vector to insert was $1: 10$, as quantified by visualization of samples of vector and insert DNA in ethidium-bromide-stained agarose gels. Restriction digests and sequencing were done to confirm the orientation and in-frame status of the inserts.

Transformation. The promoter-reporter gene constructs were transfected into Dictyostelium amoebae of the strain $\mathrm{AX} 3 \mathrm{~K}$ by using a calcium phosphate precipitation/glycerol shock protocol (Nellen et al., 1984). Transformants were selected by incubation for $6 \mathrm{~d}$ in HL5 containing $20 \mu \mathrm{g} \mathrm{G} 418$, resistance to which is encoded by the SP60-luc vector. After this selection, cells were harvested in PBS $(10 \mathrm{~mm}-\mathrm{KCl}$, $\left.10 \mathrm{~mm}-\mathrm{NaCl}, 16 \mathrm{~mm}-\mathrm{Na}_{2} \mathrm{HPO}_{4}, 34 \mathrm{~mm}-\mathrm{KH}_{2} \mathrm{PO}_{4}, \mathrm{pH} 6.5\right)$ and plated onto DM agar plates $(2 \%, \mathrm{w} / \mathrm{v}$, glucose; $1 \%, \mathrm{w} / \mathrm{v}$, Oxoid peptone; $1.5 \%$, w/v, Bacto-agar; $3 \mathrm{~mm}-\mathrm{Na}_{2} \mathrm{HPO}_{4} ; 10 \mathrm{~mm}-\mathrm{KH}_{2} \mathrm{PO}_{4}$ ) containing $40-50 \mu \mathrm{g} \mathrm{G} 418 \mathrm{ml}^{-1}$ and an overlay of neomycin-resistant $E$. coli $\mathrm{B} / \mathrm{r}$ cells (a generous gift from Dennis Welker of Utah State University, USA). Plaques appeared in $2-5 \mathrm{~d}$. Cells from individual plaques were transferred to liquid HL5 containing $5 \mu \mathrm{g} \mathrm{G} 418 \mathrm{ml}^{-1}$ and grown to a density of less than $1.0 \times 10^{6}$ cells $\mathrm{ml}^{-1}$. These cells were then used in stage studies or in CAMP shaking experiments.

Luciferase assays. Following the cAMP response experiments or experiments analysing developmental expression, extracts of transformants carrying the promoter-luciferase constructs were prepared by freeze-thaw lysis of the cells. The extracts were centrifuged for $10 \mathrm{~min}$ at $12000 \mathrm{~g}$ and $5 \mu \mathrm{l}$ of the supernatant fluid was added to $100 \mu \mathrm{l}$ of a reaction mix consisting of $0.54 \mathrm{M}$-glycylglycine ( $\mathrm{pH} 8.3$ ), $4 \mathrm{mM}-\mathrm{MgCl}_{2}$ and $2.1 \mathrm{~mm}$-ATP. Luciferase units were quantified by a Berthold luminometer (Lumat LB9501) following the addition of $100 \mu 10.6 \mathrm{~mm}$ luciferin. Specific activity is expressed as luciferase units (U) (mg protein $)^{-1}$. Protein was measured by the method of Bradford (1976).

\section{Results}

Analysis of gp-1 during Dictyostelium development and in response to exogenous $c A M P$

Fig. 1 shows a comparison of gp-1 enzyme activity, protein levels and mRNA expression during the developmental cycle of Dictyostelium. The gp-1 enzyme activity is maintained at high levels in undifferentiated amoebae, but slowly decreases during the time-course of multicellular development (Fig. 1a). Curiously, this decrease in gp-1 activity was not accompanied by a corresponding decrease in the level of gp-1 protein; gp1 protein levels remained constant during the developmental stages (Fig. $1 b$ ). In addition, Northern blot analysis showed that the gp-1 mRNA was highly expressed in vegetative cells and late in development, but decreased markedly during the initial stages of development (Fig. 1c). The patterns of both protein and mRNA expression have been observed in numerous replications and are highly reproducible. Because the observed changes in gp-1 activity do not correlate with the observed levels of gp-1 protein and mRNA, it is probable that gp- 1 is regulated at several different levels, possibly by both transcriptional and post-transcriptional events.

We have shown previously that exogenous cAMP causes a dramatic decrease in gp-1 enzyme activity (Brickey et al., 1990). Fig. 2 shows that the decrease in gp-1 enzyme activity (Fig. 2a) is not reflected in a corresponding loss of enzyme protein but, instead, gp-1 protein levels remain essentially constant in the presence or absence of exogenous cAMP (Fig. 2b). In Fig. 2(c) we have examined the effect of exogenous cAMP on gp-1 mRNA levels. Aggregation-competent Dictyostelium cells were suspended in MES/LPS buffer in either the presence or absence of $1.0 \mathrm{mM}$-cAMP. At $4 \mathrm{~h}$ and/or $8 \mathrm{~h}$ after cAMP addition cells were removed and RNA was extracted, resolved by electrophoresis, transferred to nitrocellulose filters and hybridized to radiolabelled gp1 cDNA. Essentially no gp- 1 mRNA was seen in aggregation-competent amoebae (lane 1). Cells shaken for $4 \mathrm{~h}$ or $8 \mathrm{~h}$ in the presence of cAMP (lanes 3 and 5 , respectively) showed a marked increase in the level of gp1 mRNA when compared to cells shaken in the absence of cAMP (lanes 2 and 4). These results are consistent with the hypothesis that regulation of gp-1 occurs at several levels; the changes in gp-1 activity seen in response to exogenous cAMP are not a direct consequence of regulation of the levels of gp-1 protein or gp$1 \mathrm{mRNA}$. Also, these results clearly show that the effect of exogenous cAMP on gp-1 enzyme activity, protein levels and mRNA expression is identical to that observed during normal development, suggesting that cAMP is involved in the regulation of gp-1 in vivo.

\section{Timing of $c A M P$ enhancement and the effect of $C A M P$ concentration on $\mathrm{g} p-1 \mathrm{mRNA}$ levels}

Fig. $2(c)$ shows that the cAMP enhancement of gp-1 mRNA levels appears to be maximal by $4 \mathrm{~h}$ after the addition of exogenous cAMP. We examined the kinetics 

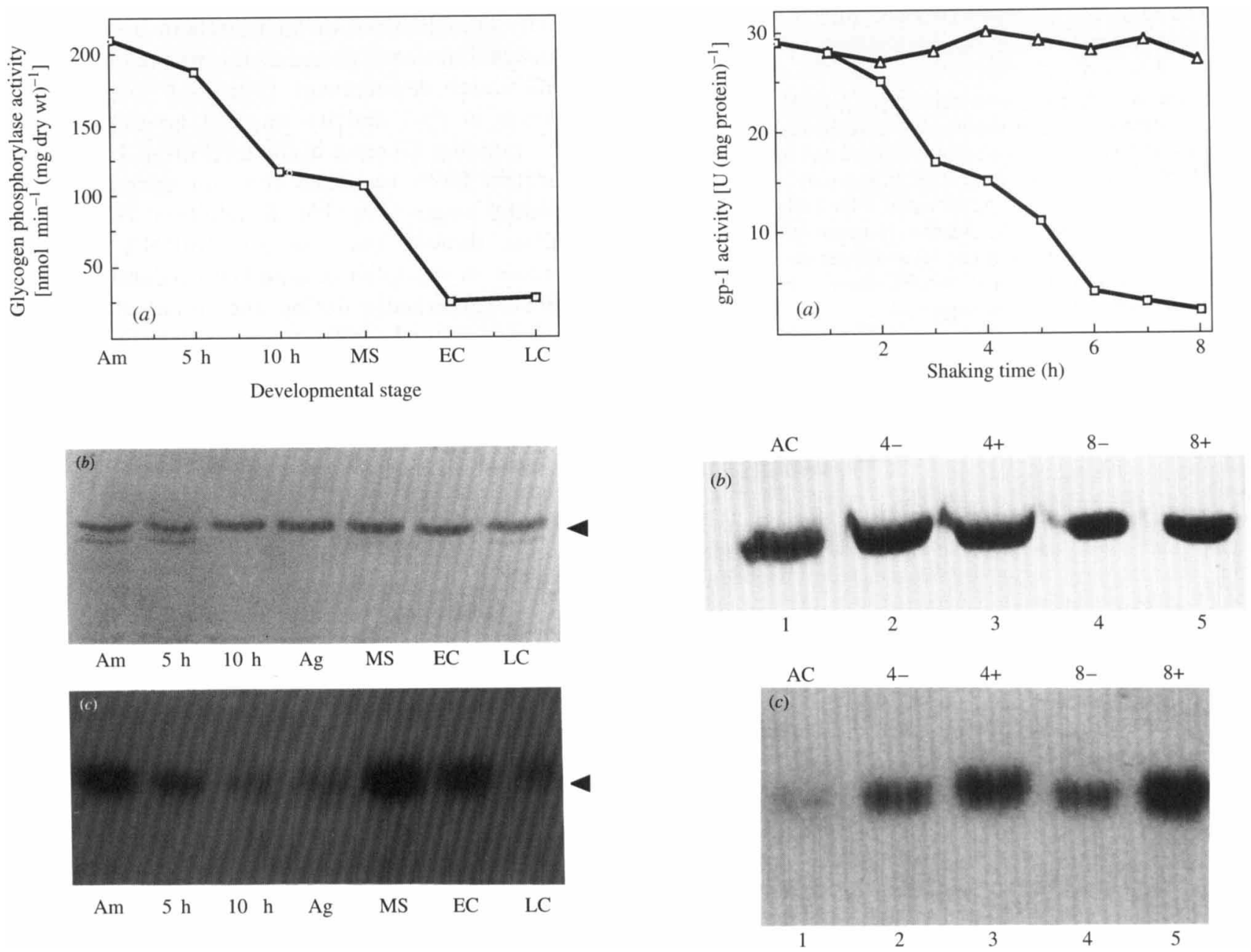

Fig. 1

Fig. 2

Fig. 1. Developmental expression of gp-1 during Dictyostelium development. (a) gp-1 activity during the development of Dictyostelium. Cells at different developmental stages were harvested, lysed, and the extracts were assayed for gp-l activity as described in Methods. Activity is expressed as nmol NADPH produced $\mathrm{min}^{-1}(\mathrm{mg} \mathrm{dry} \mathrm{wt})^{-1}$ at $23^{\circ} \mathrm{C}$. (b) Western blot showing gp-1 protein levels during Dictyostelium development. Cells at the indicated developmental stages were harvested and lysed. Proteins were resolved by SDSPAGE, transferred to nitrocellulose, and exposed to anti-gp- 1 antiserum. The arrow indicates $92 \mathrm{kDa}$, the molecular mass of purified gp-l protein. $(c)$ Northern blot analysis of gp-1 mRNA levels during the development of Dictyostelium. RNA was extracted from cells at the indicated developmental stages, resolved by agarose gel electrophoresis, transferred to nitrocellulose, and hybridized to radiolabelled gp-1 cDNA. The arrow indicates $3.0 \mathrm{~kb}$. Abbreviations: Am, vegetative amoebae; $5 \mathrm{~h}$ and $10 \mathrm{~h}$, cells plated for $5 \mathrm{~h}$ and $10 \mathrm{~h} ; \mathrm{Ag}$, late aggregation stage; MS, migrating slugs; EC, early culmination stage; LC, late culmination stage.

Fig. 2. Effects of exogenous cAMP on gp-1 enzyme activity, protein levels and mRNA levels. Aggregation-competent amoebae were resuspended in MES/LPS and shaken at 160 r.p.m. in either the presence or absence of 1 mM-cAMP. At various time-points cells were removed from the shaking cultures, lysed, and the resulting extracts were assayed for gp-1 activity or were used for Western blotting and Northern blotting. (a) gp-1 activity in cells shaken in the presence $(\square)$ and absence $(\triangle)$ of exogenous cAMP. gp-1 activity is expressed as $\mathrm{U}$ (mg protein) $)^{-1}$. (b) gp-1 protein levels in cells shaken for $4 \mathrm{~h}$ (lanes 2 and 3 ) or $8 \mathrm{~h}$ (lanes 4 and 5 ) in either the presence (lanes 3 and 5) or absence (lanes 2 and 4) of exogenous cAMP. Lane 1 (marked AC) represents protein from aggregation-competent cells. (c) gp-1 mRNA levels in cells shaken for $4 \mathrm{~h}$ (lanes 2 and 3) or $8 \mathrm{~h}$ (lanes 4 and 5) in either the absence (lanes 2 and 4 ) or presence (lanes 3 and 5) of exogenous cAMP.

of this enhancement in more detail by extracting RNA from aggregation-competent cells that had been exposed to exogenous cAMP for 1, 2, 3 or $4 \mathrm{~h}$ (Fig. $3 a$ ). The gp$1 \mathrm{mRNA}$ levels increased linearly from $1 \mathrm{~h}$ to $4 \mathrm{~h}$. Cells shaken in the absence of exogenous cAMP showed markedly less enhancement of gp-1 mRNA levels after $4 \mathrm{~h}$ of shaking (data not shown).

We also examined the effect of cAMP concentration on the enhancement of gp-1 mRNA levels (Fig. $3 b$ ). Aggregation-competent Dictyostelium cells were shaken 

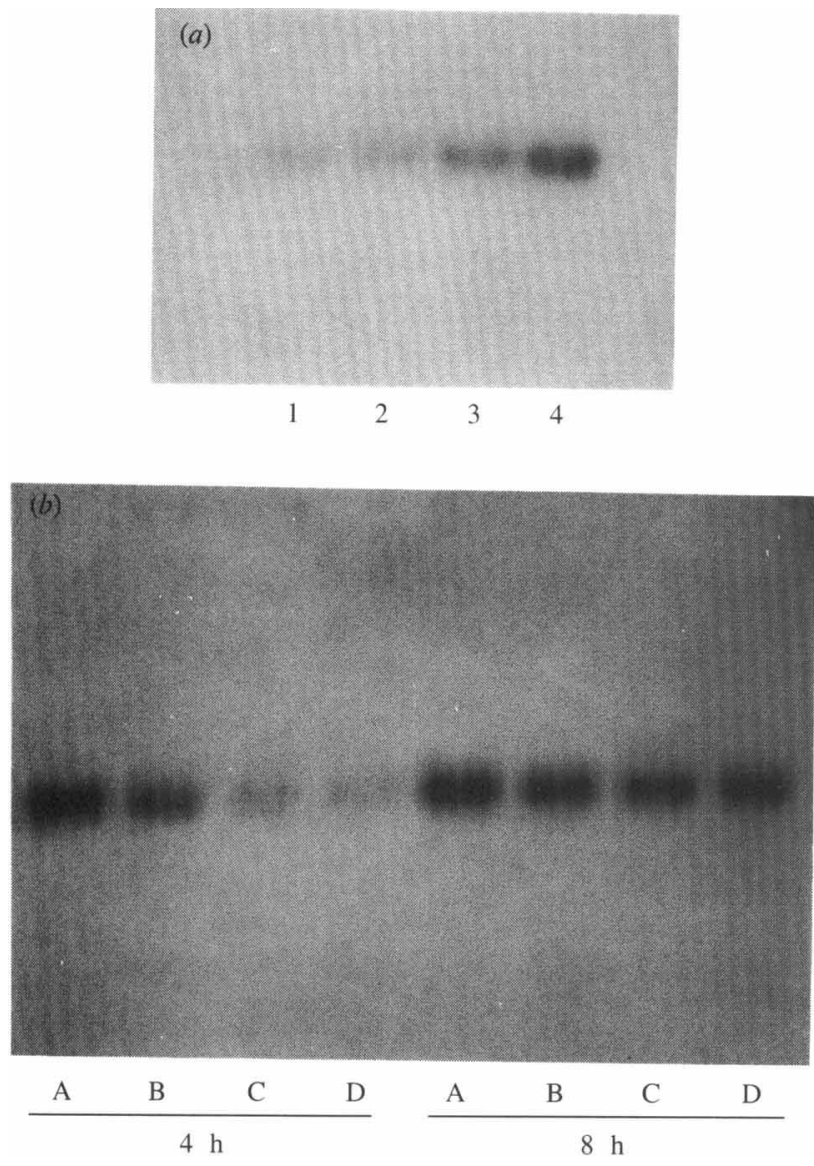

Fig. 3. Timing of cAMP enhancement and the effect of cAMP concentration on gp- 1 mRNA levels. ( $a$ ) Timing of cAMP enhancement of gp-1 mRNA levels. The Northern blot shows RNA from aggregation-competent cells that were resuspended in MES/LPS buffer containing 1.0 mM-cAMP and shaken at 160 r.p.m. for $1 \mathrm{~h}, 2 \mathrm{~h}, 3 \mathrm{~h}$ or $4 \mathrm{~h}$ (lanes 1-4, respectively). (b) Effect of cAMP concentration on gp1 mRNA levels. The RNA for this blot was extracted from aggregationcompetent cells that were shaken in the presence of cAMP at concentrations of $1.0 \mu \mathrm{M}$ (lanes D), $10 \mu \mathrm{M}$ (lanes C), $0.1 \mathrm{M}$ (lanes B), or $1.0 \mathrm{~mm}$ (lanes A) for either $4 \mathrm{~h}$ or $8 \mathrm{~h}$.

in the presence of various concentrations of exogenous cAMP for $4 \mathrm{~h}$ or $8 \mathrm{~h}$. After the incubations, RNA was extracted, resolved by electrophoresis in formaldehydecontaining agarose gels, and transferred to nitrocellulose filters. These filters were hybridized to a $836 \mathrm{bp}$ fragment of radiolabelled gp- 1 cDNA. After $4 \mathrm{~h}$ of shaking, only cAMP concentrations of $1.0 \mathrm{~mm}$ and $0.1 \mathrm{~mm}$ were effective in enhancing gp-1 mRNA levels over those expressed in the absence of cAMP. However, after $8 \mathrm{~h}$ of shaking, cAMP concentrations as low as $1.0 \mu \mathrm{M}$ resulted in elevated gp- $1 \mathrm{mRNA}$ levels. The enhancement of gp1 mRNA expression with $1.0 \mu \mathrm{M}$ occurred with cAMP concentrations $100-1000$ times lower than those routinely used to examine the regulation of Dictyostelium genes, but these concentrations are still several orders of magnitude higher than the endogenous cAMP concentration (Pahlic \& Rutherford, 1979).

\section{Molecular mechanism of cAMP enhancement of $g p-1$ expression}

The above results provide evidence that cAMP is directly involved in the regulation of gp-1 in vivo, in that exposure of cells to exogenous cAMP results in changes in the level of gp-1 enzyme activity and mRNA levels which mimic the changes that occur in normal development. In Dictyostelium, exogenous cAMP can lead to alterations in intracellular processes through at least two different molecular mechanisms (Europe-Finner \& Newell, 1987; Gerisch, 1987; Janssens \& Van Haastert, 1987; Kimmel, 1987). One mechanism involves intracellular cAMP signalling and leads to intracellular manifestations through the action of cAMP-dependent protein kinases or other cAMP binding proteins. The second mechanism is independent of intracellular cAMP signalling and appears to act through second messengers such as inositol trisphosphate $\left(\mathrm{IP}_{3}\right)$, diacylglycerol or calcium (Europe-Finner \& Newell, 1986; Schaap et al., 1986; Janssens \& Van Haastert, 1987; Europe-Finner \& Newell, 1987; Ginsburg \& Kimmel, 1989).

To examine the molecular mechanism of cAMP regulation of gp-1, aggregation-competent cells were resuspended in MES/LPS buffer in the absence or presence of cAMP as well as in MES/LPS buffer plus $2.0 \mathrm{mM}$-caffeine containing either of the cAMP analogues 2 d-cAMP or $\mathrm{Bt}_{2}$-cAMP. The selection of these analogues was based on their membrane permeability and their affinity for either the Dictyostelium cAMP-dependent protein kinase or for cell-surface cAMP receptors. Oyama \& Blumberg (1986) demonstrated that 2 'dcAMP has a high affinity for cell-surface cAMP receptors and a low affinity for the cAMP-dependent kinase, while $\mathrm{Bt}_{2}$-cAMP possesses a high affinity for the regulatory subunit of the Dictyostelium cAMP-dependent protein kinase. Also, $\mathrm{Bt}_{2}$-cAMP is membrane-permeable and is often used to mimic the effects of intracellular cAMP. Caffeine has been shown to inhibit intracellular cAMP signalling in Dictyostelium (Brenner \& Thoms, 1984). If enhancement of gp-1 mRNA expression occurs independently of intracellular cAMP signalling, under these experimental conditions only the addition of 2 'dcAMP should result in increased gp-1 mRNA levels. If, however, this enhancement is regulated via intracellular cAMP signalling, increased gp-1 mRNA levels should only be observed with $\mathrm{Bt}_{2}$-cAMP. After $4 \mathrm{~h}$ or $8 \mathrm{~h}$ incubation in the absence or presence of cAMP or the analogues, RNA was extracted and Northern blot analyses were performed. Fig. 4 shows that $\mathrm{Bt}_{2}$-cAMP (lane 4) was able to enhance the expression of gp-1 


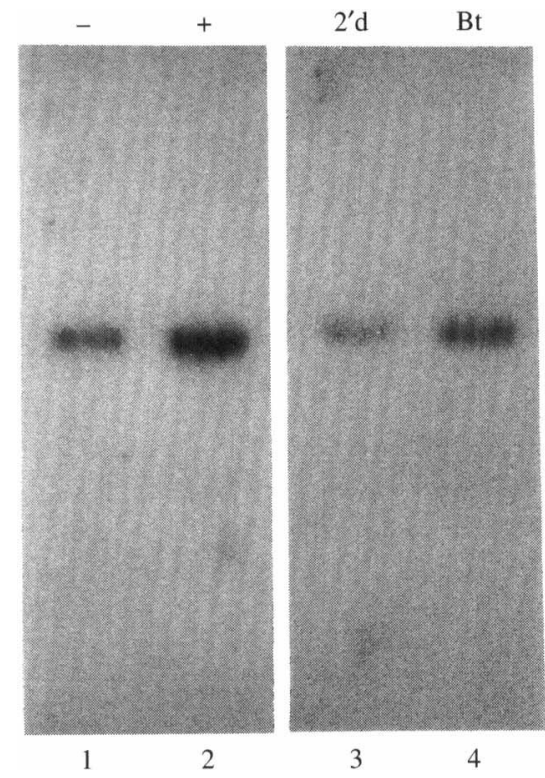

Fig. 4. Test of cAMP analogues on the enhancement of gp-1 mRNA levels. Aggregation-competent cells were shaken at 160 r.p.m. in either MES/LPS (lanes 1 and 2) or MES/LPS containing 2.0 mM-caffeine (lanes 3 and 4). Cells either received no additional treatment (lane 1) or were exposed to $1.0 \mathrm{mM}$ concentrations of cAMP (lane 2), 2' d-cAMP (lane 3) or $\mathrm{Bt}_{2}$-cAMP (lane 4). RNA was extracted after $4 \mathrm{~h}$ or $8 \mathrm{~h}$ of shaking, resolved by agarose gel electrophoresis, transferred to nitrocellulose, and hybridized to a radiolabelled gp-1 cDNA fragment.

mRNA to the levels seen with authentic cAMP (lane 2); essentially no enhancement was observed with 2 'dcAMP (lane 3). These results suggest that the cAMP regulation of gp-1 occurs through a mechanism that requires intracellular cAMP signalling.

\section{Analysis of the gp-1 promoter: identification of elements necessary for $\mathrm{g} p$-1 expression}

The complete gp-1 promoter was isolated on a $2 \cdot 2 \mathrm{~kb}$ $R s a$ I genomic fragment (Fig. 5). It is probable that this fragment contains all of the gp-1 promoter sequences because the coding sequence of another gene overlaps this fragment and deletions of this fragment confer both cAMP responsiveness and developmental regulation to a reporter gene. An open reading frame was observed beginning at nucleotide -1676 and pointing in the opposite direction from the GP1 gene. This upstream gene contained no strong sequence identity when compared to other sequences in the GenBank database. Deletion constructs of the gp-1 promoter were prepared as described in Methods and were then ligated to a luciferase reporter gene such that the gp-1 promoter directed luciferase expression. The luciferase reporter gene was derived from the vector SP60-luciferase- $\Delta 20$. The promoter deletion-luciferase constructs were then transfected into Dictyostelium amoebae. In order to

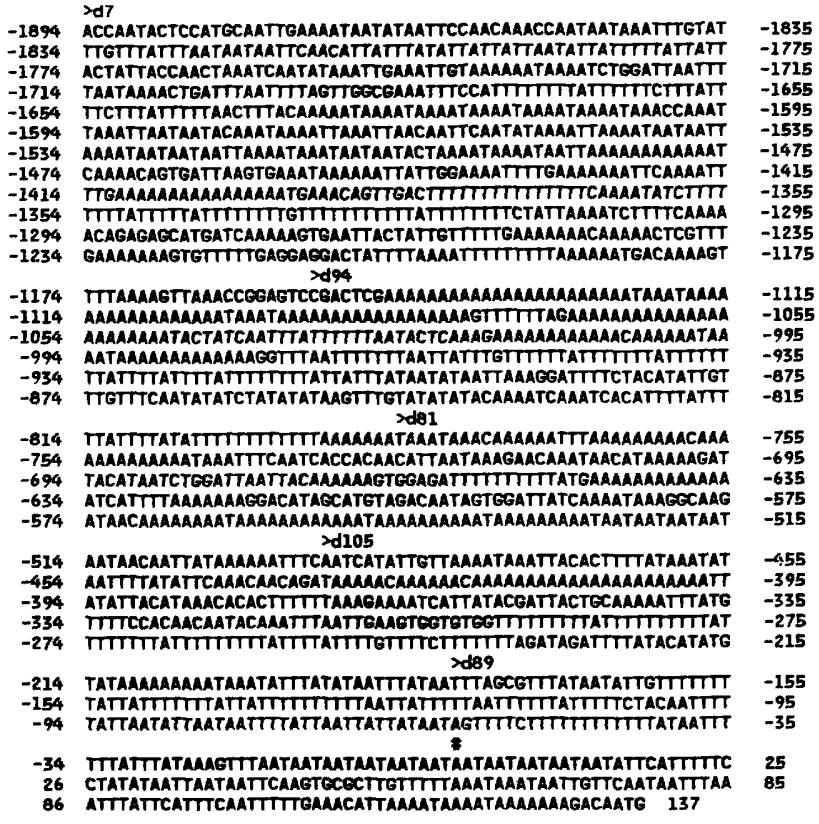

Fig. 5. Nucleotide sequence of the gp-1 promoter. The position of site Exo III deletions are indicated with the ' $>$ ' symbol over the first nucleotide in the deletion. The start site for transcription is marked by '\#'. The ATG at the end of the sequence is the amino-terminal methionine.

analyse regions of the promoter required for gp-1 expression, transformants were assayed for luciferase activity at various stages of development and after being shaken in liquid culture in the presence of exogenous cAMP.

To analyse regions of the gp-1 promoter that are required for gp-1 expression during development, transformants carrying the different promoter deletionreporter gene constructs were removed from nutrient medium and spread onto filters buffered with MES/LPS. Removal from nutrient medium triggers development; cells were harvested from the filters at various developmental stages, lysed, and the extracts were assayed for luciferase activity. Fig. 6 shows that construct gp-1d7 (extending to -1894 nucleotides, with +1 being the transcriptional start site) directed the expression of luciferase in a pattern that is the same as the developmental expression pattern observed for gp-1 mRNA (Fig. 1c); that is, high activity was observed in amoebae, followed by a decrease at the initiation of development and an increase in the late stages. Thus, expression of the luciferase reporter gene during development followed the same pattern of expression as the mRNA for the authentic GP1 gene. However, unlike the GP1 gene, the luciferase mRNA is immediately translated into enzymically active protein during both the early and late periods of increased mRNA synthesis. Conversely, the late increase in $\mathrm{gp}-1 \mathrm{mRNA}$ is not accompanied by a concomitant increase in gp- 1 enzyme 

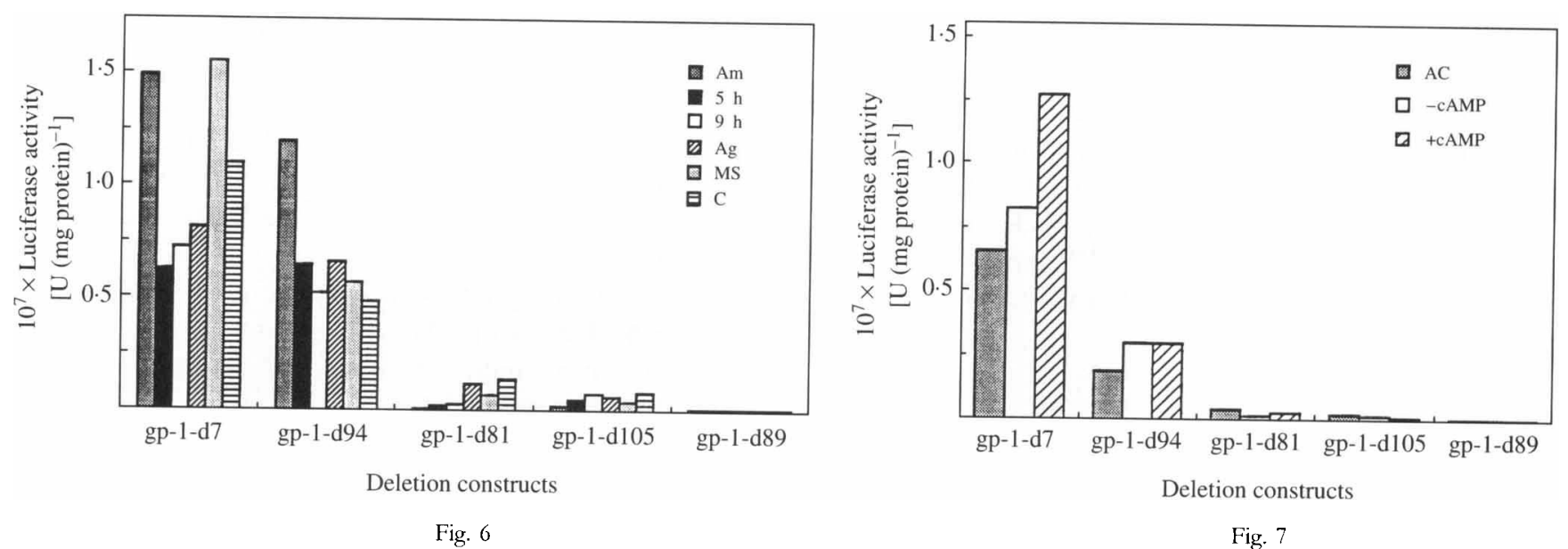

Fig. 6. Developmentally regulated luciferase activity in transformants carrying the promoter deletion-luciferase constructs. Specific activity of luciferase for each construct is shown for six developmental stages (Am, vegetative amoebae; $5 \mathrm{~h}$ and $9 \mathrm{~h}$, cells developed on filters for $5 \mathrm{~h}$ or $9 \mathrm{~h} ; \mathrm{Ag}$, late aggregation stage; MS, migrating slugs; $\mathrm{C}$, mid-culmination stage). Specific activity is expressed as $\mathrm{U}$ (mg protein $)^{-1}$.

Fig. 7. Effect of exogenous cAMP on luciferase activity in transformants carrying the promoter deletion-reporter gene constructs. Luciferase activity is shown for three experimental conditions: aggregation-competent amoebae (AC), cells shaken for $8 \mathrm{~h}$ in the absence of exogenous cAMP (-cAMP), and cells shaken for $8 \mathrm{~h}$ in the presence of $1.0 \mathrm{mM}$-cAMP (+cAMP). Luciferase activity is expressed as $U(\mathrm{mg} \text { protein })^{-1}$.

activity, even though gp- 1 protein is present. This result further suggests that gp-1 expression is regulated at both transcriptional and post-transcriptional levels. Construct gp-1-d94 (deleted to - 1153) also showed high luciferase activity, but the pattern of expression differed from gp$1-\mathrm{d} 7$ in that the late increase in activity was not seen. Constructs gp-1-d81 (-785) and gp-1-d105 (-492) showed greatly reduced luciferase activity at all stages of development, particularly in vegetative amoebae where 50-70-fold less activity was observed as compared to the longer constructs. However, the -785 and -492 constructs did appear to show a basal level of activity in the later developmental stages. Construct gp-1-d89 (deleted to - 180) showed essentially no reporter gene activity either in vegetative cells or in cells at later developmental stages. These results suggest that the alterations in gp- 1 mRNA levels during development occur as a result of transcriptional regulation, since our promoter deletions alter both the level and pattern of reporter gene expression. If, instead, gp-1 mRNA levels were controlled at the level of message stability, we would expect that promoter deletions would alter the level of expression, but the pattern of expression would remain the same for all constructs. The data presented in Fig. 6 also suggest that at least three regulatory sites exist in the gp-1 promoter. A regulatory site required for vegetative expression is located upstream of construct gp-1-d81 (-785). Luciferase expression in later developmental stages is maximal only in deletion gp-1-d7, suggesting the presence of a regulatory element necessary for late expression that is located over 1000 nucleotides upstream of the transcription initiation site. The results also suggest that there are elements able to act as a minimal promoter for late developmental activity in constructs gp-1-d105 and gp-1-d81, and elevated, but not normal, late activity in construct gp-1-d94. Note that these constructs extend from -180 to -1153 .

To analyse elements of the gp-1 promoter that are required for regulation by exogenous cAMP, the transformants carrying the promoter deletion-luciferase constructs were examined for cAMP responsiveness. Fig. 7 shows that construct gp-1-d7 $(-1894)$ resulted in a pattern of luciferase expression similar to that observed for gp-1 mRNA expression in cAMP response experiments. In both cases, the presence of exogenous cAMP enhanced the expression of luciferase or gp-1 mRNA (Fig. 7; compare to Fig. 2c) over the levels of cells shaken in the absence of cAMP. Interestingly, the specific activities of luciferase for this construct were essentially identical in these cAMP response experiments (in which cells are suspended in liquid culture) and in the experiments examining developmental expression (in which cells were plated on a solid substrate) (compare Figs 6 and 7). Construct gp-1-d94 (-1153) showed high luciferase activity but, unlike construct gp-1-d7, no enhancement of luciferase activity was seen in the presence of cAMP. Constructs gp-1-d81 and gp-1-d105 ( -785 and -492 , respectively) showed greatly reduced luciferase activity, while deletion gp-1-d89 (-180) showed no reporter activity. These results suggest that the cAMP enhancement of gp-1 mRNA levels is a transcriptional event since, again, promoter deletions 
altered both the level and pattern of expression. These results also suggest that sequence elements that are required for CAMP regulation are present only in construct gp-1-d7 and, therefore, are located at least 1153 nucleotides from the transcriptional start site. Sequences downstream from - 1153 nucleotides may, of course, also be involved in the cAMP response, but if so, require an additional sequence that is found only in the gp-1-d7 construct.

\section{Discussion}

In this report we have examined the expression of gp-1 during the development of Dictyostelium and have found evidence that the system is regulated at both the transcriptional and post-transcriptional levels. During development, gp-1 enzyme activity decreases while gp-1 protein levels remain constant. Interestingly, the gp-1 mRNA shows a pattern of expression in which high levels are observed in vegetative cells and late in development, with little or no gp-1 message seen during the intervening developmental stages. While these results clearly imply that regulation of gp-1 occurs at several levels, it is regulation by post-translational steps that control catalytic activity during development. Many specific facets of this regulation remain unknown. For example it is not clear at this point what mechanism is involved in the apparent inactivation of the gp-1 enzyme during development. Presumably, some developmentally regulated modification(s) mediate this process. It is also unclear if there is any functional role for the inactive gp1 protein that remains present throughout the late developmental stages. Preliminary information on the involvement of gp-1 enzyme in the late stages of development comes from our studies of transformed mutants which lack gp-2. In these transformed cells there is no developmental decrease in gp-1 activity, that is both gp- 1 activity and gp- 1 protein levels remain high throughout development. It is possible that the normal expression of gp-2 may be involved in regulating the activity of gp-1 (P. V. Rogers and others, unpublished). In addition, we have observed that the inactive gp-1 protein is highly localized in pre-stalk cells in Dictyostelium slugs and culminates that express gp- 2 , but in the gp- 2 transformants the active gp- 1 protein is observed in both cell types (P. V. Rogers and others, unpublished). This result suggests, again, that coordinate regulation between gp-1 and gp-2 may occur. The functional significance of an increase in gp- 1 mRNA levels during the terminal stages of development is also unclear. One possibility is that this mRNA is sequestered in spores for use upon germination, but this hypothesis has not yet been directly tested.

We have also shown that addition of exogenous
cAMP can result in changes in gp-1 enzyme activity and mRNA levels that mimic those occurring during normal development, a result that suggests that cAMP is involved in the regulation of gp-1. We have shown that the cAMP enhancement of gp-1 mRNA levels occurs in a linear fashion over $4 \mathrm{~h}$ after the initial exposure to exogenous CAMP and that exogenous cAMP concentrations as low as $1.0 \mu \mathrm{M}$ can enhance gp- $1 \mathrm{mRNA}$ levels after $8 \mathrm{~h}$ of exposure. We also examined the molecular mechanism through which cAMP regulates gp-1 expression and found evidence to support the involvement of intracellular cAMP signalling. Since cAMP has unique effects on both gp- 1 activity and gp- 1 mRNA levels, it seems likely that intracellular cAMP signalling triggers both post-translational regulation of gp- 1 as well as alterations in gp-1 transcription. Curiously, most of the other cAMP-regulated Dictyostelium genes that have been examined, including the GP2 gene in our laboratory (J. F. Sucic and others, unpublished), are regulated without intracellular cAMP signalling (Oyama \& Blumberg, 1986; Haribabu \& Dottin, 1986; Kimmel, 1987; Mann \& Firtel, 1987).

We have also examined the gp-1 promoter and identified regions which are necessary for gp-1 expression. The results of these experiments suggest that at least three regulatory sites may be present in the gp-1 promoter. Maximal vegetative expression was observed only when the region from between -785 to -1894 is not deleted from the promoter, suggesting the presence of a vegetative specific element in this region. Late developmental expression of gp- 1 was not observed if the region between -1153 and -1894 was deleted. Interestingly, this region also appears to be required for cAMP enhancement, suggesting that the cis-acting elements necessary for these aspects of gp-1 expression are located in this region of the promoter. A basal, late developmental activity was detected in constructs that extended from -180 to -785 , and elevated, but not normal, late activity was observed when the region between -785 and -1153 was included in the constructs. These results suggest the presence of elements in these regions that may be required, but are not alone sufficient, for normal late gp-1 expression.

Based on our deletion analysis, it appears that the gp1 promoter is very different from most other Dictyostelium promoters in several respects. The presence of putative $c i s$-acting elements over 1000 nucleotides from the transcriptional start site is very unusual, especially for developmental and cAMP response elements, which have usually been identified between -200 and -700 nucleotides of the transcriptional start site (Pears \& Williams, 1987; Datta \& Firtel, 1988; Early \& Williams, 1989; Pavlovic et al., 1989; Fosnaugh \& Loomis, 1991; Haberstroh et al., 1991; Faix et al., 1992; Tasaka et al., 
1992). The distance between the transcriptional start and these potential gp-1 regulatory elements is characteristic of enhancer sequences seen in higher eukaryotes (Khoury \& Gruss, 1983; Schaffner et al., 1985). Sequence analysis of the gp-1 promoter showed that several relatively $\mathrm{G} / \mathrm{C}$ rich 'boxes' are located in an otherwise extremely $\mathrm{A} / \mathrm{T}$ rich region. G/C-rich or G-rich sequences have been implicated as being involved in mediating the expression of other Dictyostelium genes that exhibit developmental and cAMP-mediated regulation (Pears \& Williams, 1987, 1988; Datta \& Firtel, 1988; Pavlovic et al., 1989; Faix et al., 1992). We are currently beginning gel-shift and mutagenesis studies to examine the potential role of these $\mathrm{G} / \mathrm{C}$-rich sequences in the regulation of gp-1.

The promoter deletion experiments also suggest that the changes in gp-1 mRNA levels that are observed during development occur as a result of transcriptional changes, rather than as a result of changes in mRNA stability, because our promoter deletions are able to alter both the level and pattern of reporter gene expression. If mRNA stability controlled gp-1 mRNA levels, we would have expected that the promoter deletions would alter the level of expression, but not the pattern of expression, in different constructs. The observation that only our longest construct directs the expression of the reporter gene in a pattern that matches the developmental pattern of gp-1 mRNA also suggests that the information required for this expression is located in the gp-1 promoter sequence contained in that construct. If nucleotide sequences in the gp-1 mRNA $5^{\prime}$ untranslated region or $3^{\prime}$ untranslated region regulated mRNA levels, we would not have expected this result, since all of the constructs contained the complete gp- $15^{\prime}$ untranslated mRNA sequence and all of them lacked any $3^{\prime}$ untranslated sequence from the gp- 1 mRNA.

We thank Kathy Lindgren, Sandra Peters-Weigel and U. R. Furball for their expert technical assistance. We also thank Ornella Selmin for helpful discussions. This work was supported by National Institutes of Health grant AG00677.

\section{References}

BRADFORD, M. (1976). A rapid and sensitive method for the quantitation of microgram quantities of protein utilizing the principle of protein-dye binding. Analytical Biochemistry 72, 248-254.

BRENNER, M. \& Thoms, S. D. (1984). Caffeine blocks activation of cyclic AMP synthesis in Dictyostelium discoideum. Developmental Biology 101, 136-146.

Brickey, D. A., Naranan, V., Sucic, J. F. \& Rutherford, C. L. (1990). Regulation of the two forms of glycogen phosphorylase by cAMP and its analogs in Dictyostelium discoideum. Molecular and Cellular Biochemistry 97, 17-33.

DatTA, S. \& FirTel, R. A. (1988). An 80-bp cis-acting regulatory region controls cAMP and development regulation of a prestalk gene in Dictyostelium. Genes and Development 2, 294-304.

Early, A. \& Williams, J. G. (1989). Identification of sequences regulating the transcription of a Dictyostelium gene selectively expressed in pre-spore cells. Nucleic Acids Research 17, 6473-6484.
Europe-Finner, G. N. \& Newell, P. C. (1986). Inositol 1,4,5, trisphosphate induces calcium release from a non-mitochondrial pool in amoebae of Dictyostelium. Biochimica et Biophysica Acta 887, 335-340.

Europe-Finner, G. N. \& Newell, P. C. (1987). Cyclic AMP stimulates accumulation of inositol trisphosphate in Dictyostelium. Journal of Cell Science 87, 221-229.

FaIX, I., Gerisch, G. \& NoEgel, A. A. (1992). Overexpression of the csA cell adhesion molecule under its own cAMP-regulated promoter impairs morphogenesis in Dictyostelium. Journal of Cell Science 102, 203-214.

Feinberg, A. P. \& Vogelstein, B. (1983). A technique for radiolabeling restriction endonuclease fragments to high specific activity. Analytical Biochemistry 132, 6-13.

FosNAUGH, K. L. \& LoOMIS, W. F. (1991). Coordinate regulation of the spore coat genes in Dictyostelium. Developmental Genetics 12, $123-132$.

Gerisch, G. (1987). Cyclic AMP and other signals controlling cell differentiation in Dictyostelium. Annual Review of Biochemistry 56, 853-879.

GinsburG, G. \& Kimmel, A. R. (1989). Inositol trisphosphate and diacylglycerol can differentially modulate gene expression in Dictyostelium. Proceedings of the National Academy of Sciences of the United States of America 86, 9332-9336.

Gustafson, G. L. \& Wright, B. E. (1972). Analysis of approaches used in studying differentiation of the cellular slime mold. Critical Reviews in Microbiology 1, 453-478.

HaberstroH, L. \& FirTel, R. A. (1990). A spatial gradient of expression of a cAMP-regulated prespore cell-type-specific gene in Dictyostelium. Genes and Development 4, 596-612.

Haberstroh, L., Galindo, J. \& Firtel, R. A. (1991). Developmental and spatial regulation of a Dictyostelium prespore gene: cis-acting elements and a cAMP induced, developmentally regulated DNA binding activity. Development 113, 947-958.

Haribabu, B. \& DotTin, R. P. (1986). Pharmacological characterization of cyclic AMP receptors mediating gene regulation in Dictyostelium discoideum. Molecular and Cellular Biology 6, 2402-2408

Janssens, P. M. W. \& Van HaAstert, P. J. M. (1987). Molecular basis of transmembrane signal transduction in Dictyostelium. Microbiological Reviews 51, 396-418.

Khoury, G. \& Gruss, P. (1983). Enhancer elements. Cell 33, 313-314.

KimMEL, A. R. (1987). Different molecular mechanisms for cAMP regulation of gene expression during Dictyostelium development. Developmental Biology 122, 163-171.

Loomis, W. F. (1982). The Development of Dictyostelium discoideum. New York: Academic Press.

MANN, S. K. O. \& FiRTEL, R. A. (1987). Cyclic AMP regulation of early gene expression in Dictyostelium discoideum: mediation via the cell surface cyclic AMP receptor. Molecular and Cellular Biology 7, 458-469.

Marshall, R., Sargent, D. \& Wright, B. E. (1970). Glycogen turnover in Dictyostelium discoideum. Biochemistry 9, 3087-3094.

Naranan, V., Sucic, J. F., Rrickey, D. A. \& Rutherford, C. L. (1988). The relationship between the two forms of glycogen phosphorylase in Dictyostelium discoideum. Differentiation 38, 1-10.

Nellen, W., Silan, C. \& Firtel, R. A. (1984). DNA-mediated transformation in Dictyostelium discoideum: regulated expression of an actin gene fusion. Molecular and Cellular Biology 4, 2890-2898.

Nellen, W., Datta, S., Reymond, C., Sivertsen, A., Mann, S., Crowley, T. \& FirTel, R. A. (1987). Molecular biology in Dictyostelium: tools and applications. Methods in Cell Biology 28, $67-108$.

Oyama, M. \& BlumberG, D. D. (1986). Interaction of cAMP with the cell surface receptor induces cell-type-specific mRNA accumulation in Dictyostelium discoideum. Proceedings of the National Academy of Sciences of the United States of America 83, 48194823.

PaHLIC, M. \& RUTHERFord, C. L. (1979). Adenylate cyclase activity and cyclic AMP levels during the development of Dictyostelium discoideum. Journal of Biological Chemistry 254, 9703-9707.

Pavlovic, J., Haribabu, B. \& Dottin, R. P. (1989). Identification of a signal transduction response sequence element necessary for in- 
duction of a Dictyostelium discoideum gene by extracellular cAMP. Molecular and Cellular Biology 9, 4660-4669.

Pears, C. J. \& Williams, J. G. (1987). Identification of a DNA sequence element required for efficient expression of a developmentally regulated and cAMP-inducible gene of Dictyostelium discoideum. EMBO Journal 6, 195-200.

Pears, C. J. \& Williams, J. G. (1988). Multiple copies of a G-rich element upstream of a cAMP inducible Dictyostelium gene are necessary but not sufficient for efficient gene expression. Nucleic Acids Research 16, 8467-8486.

Rogers, P. V., Luo, S., Sucic, J. F. \& Rutherford, C. L. (1992). Characterization and cloning of glycogen phosphorylase 1 from Dictyostelium discoideum. Biochimica et Biophysica Acta 1129, 262-272.

Rutherford, C. L. \& Cloutier, M. J. (1986). Identification of two forms of glycogen phosphorylase in Dictyostelium. Archives of Biochemistry and Biophysics 250, 435-439.

Rutherford, C. L., Peery, R. B., Sucic, J. F., Yin, Y., Rogers, P. V., Luo, S. \& Selmin, O. (1992). Cloning, structural analysis, and expression of the glycogen phosphorylase 2 gene in Dictyostelium. Journal of Biological Chemistry 267, 2294-2302.

Schaap, P., Van lookeren Campagne, M. M., Van Driel, R., Spek, W., Van HaAstert, P. J. M. \& Pinas, J. (1986). Postaggregative differentiation induction by cyclic AMP in Dictyostelium: intracellular transduction pathway and requirement for additional stimuli. Developmental Biology 118, 52-63.

SChAFFNer, W., SERfling, E. \& JASIN, M. (1985). Enhancers and eukaryotic gene transcription. Trends in Genetics 1, 224-230.

SusSman, M. \& BRACKenbURY, R. (1976). Biochemical and molecular genetic aspects of cellular slime mold development. Annual Review of Plant Physiology 27, 229-265.

Tasaka, M., Hasegawa, M., Nakata, M., OriI, H., Ozaki, T. \& TAKEUCHI, I. (1992). Protein binding and DNase-I-hypersensitive sites in the cis-acting regulatory region of the spore-coat SP96 gene of Dictyostelium. Mechanisms of Development 36, 105-115.

Wright, B. E., Simon, W. \& Walsh, B. T. (1968). A kinetic model of metabolism essential to differentiation in Dictyostelium discoideum. Proceedings of the National Academy of Sciences of the United States of America 60, 644-651. 